\title{
REVIEW
}

\section{Diagnosis and Management of Desmoid Tumors and Fibrosarcoma ${ }^{\dagger}$}

\author{
SANDRA L. WONG, MD, MS* \\ University of Michigan, 1500 E. Medical Center Drive, 3310 CCC, Ann Arbor, Michigan
}

\begin{abstract}
Fibrous tumors represent a diverse subtype of soft tissue tumors and can represent benign conditions as well as frankly malignant sarcomas. Desmoid tumors and dermatofibrosarcoma protuberans are more difficult to classify and tend to be considered in the intermediate risk category. They are distinct entities, but both are locally aggressive processes which are plagued with attendant morbidity and high recurrence rates. Complete surgical resection is the mainstay of treatment.
\end{abstract}

J. Surg. Oncol. 2008;97:554-558. (C) 2008 Wiley-Liss, Inc.

KEY WORDS: desmoid; fibrosarcoma; dermatofibrosarcoma protuberans

\section{INTRODUCTION}

Because of its distinct biologic behavior, desmoid tumors warrant separate notation in the discussion of soft tissue tumors. Desmoid tumors, or aggressive deep-seated fibromatosis, are part of a rare group of fibrous tissue proliferations which tend to be locally aggressive but have no propensity for metastasis. Desmoid tumors should be distinguished from other forms of fibromatoses such as palmar fibromatosis (Dupuytren's contracture) or fibromatosis colli (torticollis).

Desmoid tumors are uncommon and slow-growing tumors with a propensity for recurrence even after complete resection. The natural history of desmoid tumors is not well-defined and poorly understood. Clinical behavior and therefore, recommended treatments, are often dictated by anatomic site. Location of tumors can limit therapeutic options and result in significant morbidity with or without surgical resection.

\section{EPIDEMIOLOGY}

Desmoid tumors are rare, with an estimated incidence rate of two to four cases per million per year. Few centers have sufficient experience with desmoids [1-4], and there are precious little prospective cohorts which collect data and treat patients in a standard fashion. Desmoid tumors are classically described as an abdominal wall tumor, commonly seen in young women during the post-partum period [5]. However, desmoids can occur at any site in the body. Commonly, three main anatomic sites are described: (1) trunk or extremity, (2) abdominal wall, and (3) intra-abdominal (bowel and mesentery). They occur in a wide age range, between 16 and 79 years of age, but the median age is 35 , with the majority being diagnosed before the age of 40. Desmoids are slightly more common in women than men [1], but there are no apparent differences by race.

Fibromatosis is usually seen sporadically, but can be associated with familial adenomatous polyposis (FAP). Indeed, intra-abdominal and extremity desmoids represent common extracolonic manifestations of disease in Gardner's syndrome. FAP, characterized by mutation of the adenomatous polyposis coli (APC) tumor suppressor gene, has a clinical manifestation of hundreds, if not thousands, of adenomatous polyps in the colon and rectum. Proctocolectomy is performed to treat or prevent colon cancer, which had previously been the most common cause of death in FAP patients. Interestingly, mortality in this population following proctocolectomy is still higher than the general population, in part due to desmoid tumors and their resultant complications [6].

Desmoid tumors have also been associated with high estrogen states and episodes of antecedent trauma, but the evidence is largely based on retrospective and anecdotal reports. Many of the reports with an inciting traumatic event include "trauma" from a prior operation if desmoids occurred at an incision site, particularly when prior operations were performed for FAP $[7,8]$. While there is a higher incidence of abdominal wall tumors in the peripartum period or with use of oral contraceptives, the link between estrogen levels and desmoid tumors is not firmly established [1]. Subsequent pregnancy in women with a pregnancy-related desmoid has not been reported as a risk factor for recurrent disease [9].

\section{HISTOPATHOLOGY AND MOLECULAR GENETICS}

Desmoids are characterized by a monoclonal fibroblastic proliferation typically arising from muscular or aponeurotic structures. On gross examination, the tumors appear firm and smooth, without necrosis or hemorrhage. An intact capsule surrounds the periphery with initial inspection, however, the tumor characteristically extends beyond this pseudo-capsule, with fibrous septae of tumor extending radially. Microscopically, there is usually a spindle-shaped pattern of

${ }^{\dagger}$ This article was intended for Seminars in Surgical Oncology: Orphan Sarcomas.

*Correspondence to: Sandra L. Wong, MD, MS, Assistant Professor of Surgery, University of Michigan, 1500 E. Medical Center Drive, 3310 CCC, Ann Arbor, MI 48109-0932. Fax: 734-647-9647.

E-mail:wongsl@umich.edu

Received 11 December 2007; Accepted 13 December 2007

DOI 10.1002/jso.20981

Published online in Wiley InterScience(www.interscience.wiley.com). 
cells, separated by thick collagen fibers and without pleomorphic nuclear staining. Immunohistochemistry are helpful with diagnosis: spindle cells usually stain for vimentin and smooth muscle actin, but are generally negative for desmin, cytokeratins, and S-100. The infiltrative fibroconnective tissue process which characterizes desmoid tumors may resemble that of low-grade fibrosarcoma, but their histologic appearance lacks nuclear and cytoplasmic features of malignancy. There features also do not support any metastatic potential of these benign appearing tumors.

Some had previously classified desmoid tumors as an unchecked reactive process rather than a neoplastic process due to its lack of mitotic activity. However, desmoids show uniform patterns of Xchromosome inactivation, confirming tumors of clonal composition [10]. Interestingly, recent data demonstrate a molecular connection between wound healing processes and fibroproliferative disorders of mesenchymal tissue. Inducible transgenic expression of a mutant form of the beta-catenin gene (CTNNB1) produces hyperplastic wound healing responses, and somatic mutations in codons 41 or 45 of exon 3 of CTNNB1 have been linked to sporadic desmoid tumors $[11,12]$.

Overall, approximately $2 \%$ of desmoids are associated with FAP, but desmoids are seen in about $10-15 \%$ of FAP cases, making these patients at 1,000-fold risk of desmoids compared to the general population. Because of this genetic predisposition to desmoid tumors in FAP patients, mutation analyses of the APC gene may help elucidate the pathogenesis of fibromatosis. However, current evidence demonstrates that there are likely undefined genetic or clinical factors independent of APC which are responsible for fibromatosis though location of the mutation $\left(3^{\prime}\right.$ (distal) to codon 1399) does appear to be associated with some increased risk for desmoids [13,14].

Because CTNNB1 and APC are both part of the Wnt signaling pathway, mutations in either gene result in beta-catenin protein stabilization and subsequent downstream activation of the T-cell factor/ lymphoid enhancer factor (TCF/Lef) family of transcription factors [12]. Resultant activation of this pathway may be a potential target for future study and development of tailored therapies.

Other molecular studies which have generated some interest include findings of the differential expression of estrogen receptor subtypes, $\alpha$ and $\beta$, in desmoid tumors. There appears to be an increased estrogen receptor- $\beta$, but not estrogen receptor- $\alpha$, expression in $80 \%$ of desmoid specimens [15]. In fact, studies show that no estrogen receptor- $\alpha$ expression was seen most specimens. Increased expression of plateletderived growth factor receptors (PDGFR, A and B) and their ligands in desmoid tumors has also been seen [16]. These findings lend some support for the treatment of tumors with adjuvant agents such as tamoxifen and imatinib, respectively.

\section{CLINICAL FEATURES AND TREATMENT}

\section{Surgical Resection}

Desmoid tumors may arise at any site in the body, but are most commonly found in the trunk and extremities. In these locations, tumors tend to be located in deep in the muscles or along fascial planes, such as at a point of muscular insertion. Patients will usually present with a greater than $5 \mathrm{~cm}$, localized, firm mass with an indolent pattern of growth, which can be painless or minimally painful. Those tumors of the abdominal wall occur along the anterior abdominal wall, most commonly in young women during or immediately after pregnancy. Intra-abdominal presentations can be associated with mass effect, intestinal obstruction, or mucosal ischemia. FAP patients often present with concomitant intra-abdominal and abdominal wall disease [17]. Other well-characterized, though rare, sites include the head and neck region [18] and the breast [19].
In all locations, desmoid tumors are notoriously infiltrative and persistent. Site may not be related to a biologically significant difference, but the locally aggressive nature of desmoid tumors renders site the major factor in treatment planning and management strategies. Complete resection is considered the best course of initial treatment and is potentially curative. Ability to accomplish complete resection at the first attempt defines likelihood of recurrence, so it is important to have a sufficient preoperative suspicion of fibromatosis and to be circumspect in surgical technique. Initial diagnosis with core needle biopsy may be helpful in planning of a radical resection.

Clinical evaluation should be complemented with radiologic studies. Cross-sectional imaging with CT scans or MRI should be performed in most cases to determine proximity to adjacent structures. While there are no pathognomonic characteristics of desmoids over other soft tissue tumors, imaging studies, particularly MRI, provide excellent definition of pattern and extent of involvement when proper clinical correlation is made. There is no need for staging studies because there is no propensity for regional or distant disease.

Resection does not appear to affect survival, not a surprising finding given the histologically benign nature of desmoid tumors. Desmoid tumors have a propensity for recurrent disease, and it is unclear if incomplete resection contributes to the local recurrence rate [3,17-20]. While complete extirpation of tumor with negative microscopic margins is the standard surgical goal, this accomplishment is often constrained by anatomic boundaries and not necessarily an assurance of cure. Positive margins are seen in a significant portion of patients [1,3]. However, positive margin status does not inevitably lead to recurrent disease, just as patients with a complete microscopic resection are found to have local recurrences. Time to recurrence has been reported at a median time of 18 months, but ranges from 4 months to almost 12 years [20]. Clinicopathologic factors such as age, gender, depth of tumor, specific tumor site, and margin status were not predictive of risk for local recurrence in extremity and truncal desmoids [20].

In extremity desmoids, involvement of deep muscular bundles or neurovascular structures could limit limb-sparing options. The extent of surgical resection is somewhat controversial, however, as aggressive attempts (e.g., amputation) at achieving a negative margin can result in unnecessary morbidity [1].

For intra-abdominal tumors, extensive association with the mesentery limits the extent of resection and could predispose to significant morbidity, including complications of bowel ischemia, adhesions and resultant obstruction, and even fistulae formation [17]. Because the tumor biology is notoriously unpredictable, periods of rapid tumor growth can be followed by stability or even regression. Heroic resections could lead to short gut syndrome, and while small bowel transplantation has been considered in the past, its use is limited to very select cases [21]. Many extensive cases of mesenteric fibromatosis are technically unresectable and can result in worsening symptoms of bowel obstruction and ischemia. Ultimately, there can be fatal outcomes over time.

Desmoid tumors of the breast are an unusual but distinct entity. Importantly, they tend to present as palpable masses suspicious for carcinoma both clinically and radiographically. Primary therapy remains primarily surgical, and can be considered curative. As with other locations, prediction of recurrent cannot reliably be made from margin status alone. As many as $16 \%$ of patients with negative margins experienced recurrences over a 25 -year study period, so clinical judgment should dictate extent of resection and reresection [19].

Patients with fibromatosis of the head and neck region are also plagued with the constraints of complex anatomy and particularly the proximity of critical structures. Many patients have neurologic dysfunction, including severe pain or radiculopathy, at time of presentation and good functional outcomes are limited, with over $60 \%$ of patients reporting motor deficits or paresthesias [18]. 
As adjuvant treatments for involved margins or alternate treatments to better preserve function in challenging anatomic locations, systemic therapies and radiotherapy have been considered and increasingly utilized.

\section{Radiation Therapy}

There are no strong data to support the use of radiation therapy in the adjuvant setting following complete surgical resection. The utility of radiation in the setting of positive resection margins is thought to be quite minimal given the difficulty in predicting risk of recurrence in these patients. While there was some initial enthusiasm for postoperative radiation for tumors with involved margins because of improved recurrence rates [22,23], many series have failed to report any improvement in recurrence rates [20,24]. Though highly controversial, its use is reasonably deferred until recurrence is seen and not amenable to repeat resection $[3,20]$ unless a recurrence were to result in intractable morbidity.

Toxicity to surrounding tissue and potential for late radiation effects such as secondary malignancies must be considered in the decisionmaking process. Typical doses of external beam radiation treatments are approximately 50-56 Gy, delivered at 1.8 Gy per fraction once daily. The use of radiation therapy in lieu of surgical resection has been discussed, but not uniformly applied. In the scenario of unresectable intra-abdominal disease, use of radiation is also limited because of bowel toxicity. Because palliative resection is not felt to yield improvement in symptoms or lengthen survival, there should be consideration of radiation therapy in truncal or extremity desmoids when surgical options are no longer feasible. Reported results show good local control rates in select situation.

The use of radiation in the preoperative setting, with or without systemic treatments, has only been explored in single-institution settings [4], but these approaches have not been widely tested in large numbers of patients. One approach entails the use of neoadjuvant doxorubicin with radiotherapy, followed by surgical resection for lesions which were felt to be resectable upon presentation. Local control rates were good, even for recurrent disease, but it was a small study and confirmatory data are not yet available.

\section{Systemic Treatments}

Use of systemic therapies is enticing for patients with recurrent disease or patients with unresectable lesions. A number of treatment regimens have been used in the past and in present practice with varying results. Chemotherapy, whether administered as a single agent or as a combination therapy, is met with skepticism since oncologic dogma dictates that cytotoxic treatments are not particularly effective in indolent tumors without metastatic potential. Its use is uncommon, ranging from none to approximately $25 \%[3,20,23,24]$ in several large series of patients. Nevertheless, agents such as methotrexate, doxorubicin, dacarbazine, vinblastine, and vinorelbine have shown some activity in very select populations $[25,26]$, particularly in FAP patients.

Noncytotoxic agents which have been used for systemic treatment of desmoid tumors can be broadly categorized: hormonal agents, nonsteroidal anti-inflammatory agents, and targeted molecular agents. Because of clinical and experimental evidence linking hormonal surges to desmoid growth $[1,15]$, anti-estrogen agents (tamoxifen, selective estrogen receptor modulators (SERMs)) have been commonly used over the years. While occasionally prescribed in a prophylactic setting, its main indication is for disease which fails other means of local control. Tamoxifen is the most commonly prescribed, though newer drugs such as toremifene and raloxifene have also been used. Interestingly, the present of estrogen receptor- $\alpha$ is not reliably predictive of response, but newer evidence suggests a role for estrogen receptor- $\beta$ which is commonly seen in desmoid tumors [15]. However, even in the best of circumstances, $50 \%$ of patients have partial responses with either tumor shrinkage or stabilization of disease $[27,28]$ and responses are sometimes seen after the medication is discontinued.

There have been some responses to non-steroidal anti-inflammatory agents as well. Sulindac has been most commonly prescribed, and it is sometimes given in combination with tamoxifen. The most success has been seen in selected FAP patients [29], but most series only show partial regression or stabilization of disease, and duration of response is not well reported. True anti-fibrotic agents have been used outside of the United States, but represents a very limited experience. Trials of interferon have also been reported in the past.

The recent use of the tyrosine kinase inhibitor imatinib has generated some enthusiasm, though the mechanism of action remains unclear [16,30]. Tissue analyses have not uniformly demonstrated activated c-kit or PDGFRRA receptors, though studies are limited in size. Because of ongoing research and recent findings with the Wnt/ beta-catenin signaling pathway in the pathogenesis of desmoid tumors, other novel molecularly targeted agents may be in development soon [12].

In general, the use of pharmacologic agents, is not widely accepted except in cases where other options are not possible. While some of the noncytotoxic agents have a relatively low side effect profile, the riskbenefit ratio has yet to be defined. Enthusiasm is hampered by reports of partial responses with either minimal tumor shrinkage or stabilization of disease as endpoints in a disease process where the natural history is unpredictable to begin with.

\section{SURVEILLANCE}

The potentially long natural history of desmoids makes reliable documentation of long-term results difficult. Locally recurrent desmoids can be difficult to control. Those with recurrent disease do have a greater risk of subsequent recurrence. Local recurrence rates are site-dependent, with a more favorable prognosis for abdominal wall sites. There are no standardized protocols for surveillance, but most patients are followed with clinical examination and radiographic studies (depending on anatomic site) at biannual or annual intervals.

Recurrent disease should be considered for resection when feasible. Aggressive resection for recurrent disease must be balanced against resultant morbidity, which can be quite severe. Radiation or systemic options can be considered if resection is not possible or would result in undue functional or cosmetic outcome. Observation can also be considered if patients are relatively asymptomatic.

\section{FIBROSARCOMA}

While there is no risk of degeneration from fibromatosis to fibrosarcoma, this is a distinct type of soft tissue sarcoma. Unfortunately, there are no distinct clinical characteristics of fibrosarcoma, and disease is poorly described in the context of all fibroblastic tumors.

Classic fibrosarcomas are seen in persons aged 30-55 years. Fibrosarcomas can be part of the spectrum of radiation-induced or radiation-associated sarcomas, representing about $15 \%$ of the histologic subtypes [31]. They are characterized by elongated, fibroblast-like cells that grow in a uniform, fasciculated pattern. Well-differentiated fibrosarcomas are rich in mature collagen. Treatment options are dictated by location, and surgical resection remains the mainstay of curative therapy.

Dermatofibrosarcoma protuberans (DFSP) is a specific form of soft tissue sarcoma which is nearly always considered a low-grade sarcoma [32]. These tumors are prone to recurrence, with high local recurrence 
rates of up to $60 \%$ following resection. Occasionally, DFSP will undergo transformation to classic fibrosarcoma. A fibrosarcomatous variant of DFSP has been described and is associated with a decreased recurrence-free survival rate [33]. Risk of metastasis is low, but can occur to lung or regional lymph nodes. When distant disease is seen, fibrosarcomatous degeneration should be considered.

DFSP can occur anywhere in the body, but are seen on the trunk in about half of all cases [32]. Classically, these tumors present as a nodular cutaneous mass. The growth pattern is indolent, with slow and persistent enlargement over many years. As the lesion enlarges, it can become protuberant in nature. They are locally aggressive and associated with the ability to be invasive.

On histologic evaluation, DFSP can be confused with benign fibrous histiocytoma, but tends to grow in a more infiltrative pattern, with subcutaneous (or deeper) tissue spread. Diagnosis can be made with CD34 staining, and more than $90 \%$ of cases are associated with a chromosomal translocation involving the COL1A1 gene on chromosome 17 and the platelet-derived growth factor B gene on chromosome 22 [34]. Fibrosarcomatous change is characterized by a fascicular, herringbone pattern of growth, similar to that seen in classic fibrosarcomas. Important poor pathologic features include size, invasion of neuromuscular structures, increased cellularity, necrosis, nuclear pleomorphism, and higher mitotic index [32].

The mainstay of therapy is surgical resection. Because of their infiltrative nature, clear margins can be difficult to obtain with resection of DFSP. Tentacle-like projections from the visible tumor appear attenuated and can be mistaken for normal collagen. Involvement of margins, either frankly positive or "very close" $(<1 \mathrm{~mm})$, is an adverse prognostic factor. Up to $21 \%$ of patients develop a recurrence at a median time of 32 months. Metastatic disease is associated with a poor prognosis.

An aggressive approach to surgical resection may be necessary, but can be associated with a certain degree of morbidity. Because adequate wide margins can be difficult to achieve, Mohs micrographic surgical techniques have been advocated for DFSP. Because some lesions are quite large at time of resection, use of Mohs may be prohibitive. A modified "square technique" has been used with success in ascertaining negative margins prior to a staged closure [35] with excellent local control rates. Repeat resection should be offered for recurrent disease because of the risk of degeneration to a more aggressive disease process.

Because of the high risk of recurrence, adjunct treatment with radiation has been suggested [36,37] for patients with microscopic residual disease. While results are promising, long term control is difficult to assess. Because of the high rate of PDGFR expression in DFSP, other adjuvant therapies include imatinib, which has been used in locally advanced cases where surgical resection is not possible [34].

\section{SUMMARY}

Fibrous tumors represent a diverse subtype of soft tissue tumors. In particular, desmoid tumors and dermatofibrosarcoma protuberans are locally aggressive processes which are plagued with high recurrence rates. Complete surgical resection is the mainstay of treatment.

\section{REFERENCES}

1. Lewis JJ, Boland PJ, Leung DH, et al.: The enigma of desmoid tumors. Ann Surg 1999;229:866-872.

2. Posner MC, Shiu MH, Newsome JL, et al.: The desmoid tumor: Not a benign disease. Arch Surg 1989;124:191-196.

3. Lev D, Kotilingam D, Wei C, et al.: Optimizing treatment of desmoid tumors. J Clin Oncol 2007;25:1785-1791.

4. Baliski CR, Temple WJ, Arthur K, et al.: Desmoid tumors: A novel approach for local control. J Surg Oncol 2002;80:96-99.
5. Gansar GF, Markowitz IP, Cerise EJ: Thirty years of experience with desmoid tumors at Charity Hospital. Am Surg 1987;53:318 319.

6. Latchford AR, Sturt NJH, Neale K, et al.: A 10-year review of surgery for desmoid disease associated with familial adenomatous polyposis. Br J Surg 2006;93:1258-1264.

7. Lopez R, Kemalyan N, Moseley HS, et al.: Problems in diagnosis and management of desmoid tumors. Am J Surg 1990;159:450 453.

8. Clark SK, Phillips RK: Desmoids in familial adenomatous polyposis. Br J Surg 1996;83:1494-1504.

9. Way JC, Culham BA: Desmoid tumor. The risk of recurrent or new disease with subsequent pregnancy: A case report. Can J Surg 1999;42:51-54.

10. Li M, Cordon-Cardo C, Gerald WL, et al.: Desmoid fibromatosis is a clonal process. Hum Pathol 1996;27:939-943.

11. Cheon SS, Cheah AY, Turley S, et al.: beta-Catenin stabilization dysregulates mesenchymal cell proliferation, motility, and invasiveness and causes aggressive fibromatosis and hyperplastic cutaneous wounds. Proc Natl Acad Sci USA 2002;99:69736978

12. Kotiligam D, Lazar AJF, Pollock RE, et al.: Desmoid tumor: A disease opportune for molecular insights. Histol Histopathol 2008;23:117-126.

13. Sturt NJ, Gallagher MC, Bassett P, et al.: Evidence for genetic predisposition to desmoid tumours in familial adenomatous polyposis independent of the germline APC mutation. Gut 2004;53: $1832-1836$.

14. Durno C, Monga N, Bapat B, et al.: Does early colectomy increase desmoid risk in familial adenomatous polyposis? Clin Gastroenterol Hepatol 2007;5:1190-1194.

15. Deyrup AT, Tretikova M, Montag AG: Estrogen receptor- $\beta$ expression in extraabdomnal fibromatoses: An analysis of 40 cases. Cancer 2006;106:208-213.

16. Heinrich MC, McArthur GA, Demetri GD, et al.: Clinical and molecular studies of the effect of Imatinib on advanced aggressive fibromatosis (desmoid tumor). J Clin Oncol 2006;24:1195-1203.

17. Smith AJ, Lewis JJ, Merchant NB, et al.: Surgical management of intra-abdominal desmoid tumors. Br J Surg 2000;87:608-613.

18. Hoos A, Lewis JJ, Urist MJ, et al.: Desmoid tumors of the head and neck-A clinical study of a rare entity. Head Neck 2000;22:814-821.

19. Neuman HB, Brogi E, Ebrahim A, et al.: Desmoid tumors (fibromatoses) of the breast: A 25-year experience. Ann Surg Oncol 2007 (epub). DOI: 10.1245/s10434-007-9580-8

20. Merchant NB, Lewis JJ, Woodruff JM, et al.: Extremity and trunk desmoid tumors: A multifactorial analysis of outcome. Cancer 1999;86:2045-2052.

21. Grant D: Current results of intestinal transplantation. The International Intestinal Transplant Registry. Lancet 1996;347: 1801-1803.

22. Ballo MT, Zagars GK, Pollack A: Radiation therapy in the management of desmoid tumors. Int J Radiat Oncol Biol Phys 1998;42:1007-1014.

23. Spear MA, Jennings LC, Mankin HJ, et al.: Individualizing management of aggressive fibromatoses. Int J Radiat Oncol Biol Phys 1998;40:637-645.

24. Gronchi A, Casali PG, Mariani L, et al.: Quality of surgery and outcome in extra-abdominal aggressive firomatosis: A series of patients surgically treated at a single institution. J Clin Oncol 2003;21:1390-1397.

25. Patel SR, Evans HL, Benjamin RS: Combination chemotherapy in adult desmoid tumors. Cancer 1993;72:3244-3247.

26. Gega M, Yanagi H, Yoshikawa R, et al.: Successful chemotherapeutic modality of doxorubin plus dacarbazine for the treatment of desmoid tumors in association with familial adenomatous polyposis. J Clin Oncol 2006;24:102-105.

27. Janinis J, Patriki M, Vini L, et al.: The pharmacological treatment of aggressive fibromatosis: A systemic review. Ann Oncol 2003; 14:181-190

28. Mendenhall WM, Zlotecki RA, Morris CG, et al.: Aggressive fibromatosis. Am J Clin Oncol 2005;28:211-215. 
29. Hansmann A, Adolph C, Vogel T, et al.: High dose tamoxifen and sulindac as first-line treatment for desmoid tumors. Cancer 2004; 100:612-620.

30. Mace J, Biermann JS, Sondak V, et al.: Response of extraabdominal desmoid tumors to therapy with imatinib mesylate. Cancer 2002;95:2373-2379.

31. Cha C, Antonescu CR, Quan ML, et al.: Long-term results with resection of radiation-induced soft tissue sarcomas. Ann Surg 2004;239:903-910.

32. Bowne WB, Antonescu CR, Leung DHY, et al.: Dermatofibrosarcoma protuberans: A clinicopathologic analysis of patients treated and followed at a single institution. Cancer 2000;88: $2711-2720$

33. Mentzel T, Beham A, Katenkamp D, et al.: Fibrosarcomatous ("high grade") dermatofibrosarcoma protuberans: Clincopatho- logic and immunohistochemical study of a series of 41 cases with emphasis on prognostic significance. Am J Surg Pathol 1998; 22:576-587.

34. McArthur GA: Molecular targeting of dermatofibrosarcoma protuberans: A new approach to a surgical disease. J Natl Compr Canc Netw 2007;5:557-562.

35. Dubay D, Cimmino V, Lowe L, et al.: Low recurrence rate after surgery for dermatofibrosarcoma protuberans: A multidisciplinary approach from a single institution. Cancer 2004;100:1008-1016.

36. Suit H, Spiro I, Mankin HJ, et al.: Radiation in management of patients with dermatofibrosarcoma protuberans. J Clin Oncol 1996;14:2365-2369.

37. Ballo MT, Zagars GK, Pisters P, et al.: The role of radiation therapy in the management of dermatofibrosarcoma protuberans. Int J Radiat Oncol Biol Phys 1998;40:823-827. 